\title{
A Formulation of Quantum Theory Based on Two Physical Principles
}

\author{
R. T. Deck \\ Department of Physics, University of Toledo, Toledo, USA \\ Email: rtd@physics.utoledo.edu
}

Received 2 November 2014; accepted 21 March 2015; published 25 March 2015

Copyright (C) 2015 by author and Scientific Research Publishing Inc.

This work is licensed under the Creative Commons Attribution International License (CC BY). http://creativecommons.org/licenses/by/4.0/

(c) (i) Open Access

\begin{abstract}
We demonstrate two points: 1) the formalism of quantum mechanics can be understood simply as a structure for the expression of the physical notion that not all observables can have values simultaneously; 2) the specific uncertainty relations can be derived (rigorously) by combination of the invariance principle with a general uncertainty relation based only on the existence of unspecified pairs of conjugate observables. For this purpose, we present a formulation of quantum mechanics based strictly on the invariance principle and a "weak" statement of the uncertainty principle that asserts only the existence of incompatible (conjugate) observables without specifying which observables are incompatible. We go on to show that the invariance principle can be used to develop the equations of motion of the theory, including the Klein-Gordon and Schrodinger equations.
\end{abstract}

\section{Keywords}

Uncertainty Principle, Invariance Principle, Klein-Gordon Equation, Schrodinger Equation

\section{Introduction}

While the famous uncertainty relation $\Delta x \Delta p_{x} \geq \hbar / 2$ first stated by Werner Heisenberg is well known, few know that this relation can be derived from the translational symmetry of space on the basis of a much more general uncertainty relation. The purpose here is to indicate that the entire structure of the quantum theory and its most basic content derive from only two physical principles, which can be referred to as the principle of uncertainty and the principle of invariance (stated below). A more complete demonstration can be found in [1].

Here, by the principle of uncertainty is meant simply the notion that there are sets of incompatible observables that cannot have precise values simultaneously. More particularly, the principle expresses the fact that there exist certain pairs of "conjugate observables", $\alpha$ and $\beta$, the simultaneous values of which have irreducible intrinsic 
uncertainties $\Delta \alpha$ and $\Delta \beta$ that satisfy an inequality of the form

$$
\Delta \alpha \Delta \beta \geq \hbar / 2
$$

where $\hbar$ is a fundamental constant of nature (equal to Planck's constant $h$ over $2 \pi$ ). By the principle of invariance is meant the notion that the laws of physics should have the same form in all physically equivalent coordinate frames. It is remarkable that the entire content of ordinary (single particle) quantum mechanics can be reduced to the above two principles with no stronger statement of the principle of uncertainty than is given above. In particular, although the principle as stated asserts only the existence of pairs of observables without defining which observables are in fact conjugate, specific pairs of conjugate observables can be deduced from the general principle in the form above by combining it with the principle of invariance. More precisely, given the assumption of the existence of conjugate observables satisfying the "uncertainty relation" written above, without specifying the nature of these observables, it can be shown (in the manner indicated here) that the invariance principle requires certain pairs of observables to satisfy such a relation, and that these pairs of observables are those found in nature.

Although the manner in which quantum theory is developed is hallowed by tradition, and readers steeped in a view of the subject they have come to terms with may not be open to an alternative interpretation, it is the contention here that the development outlined below simplifies the interpretation of the theory, and therefore deserves a place in the literature. Specifically, it addresses the first question that a student of quantum mechanics should have, namely, the question why the formalism of the theory must be changed from the formalism of classical physics in which observables are represented by mathematical variables. The historical approach to quantum mechanics provides a somewhat complicated answer to this question, whereas it is made evident here that the simple answer is the existence of pairs of observables that cannot have precise values simultaneously. Although the importance of the principles of uncertainty and invariance in quantum theory is well recognized, we are unaware of either a treatise on the subject that develops the theory strictly on the basis of these two principles or a place in the literature where the two points in the abstract are stated. Instead, the theory is either presented following the line of its historical development on the basis of the asserted wave nature of matter [2], or developed (in more "rigorous" presentations) as the consequence of postulated axioms (the number and form of which can vary in different formulations) [3] [4]. In the more rigorous developments based on postulated axioms, because certain axioms represent purely mathematical statements relating to the structure of the formalism while other axioms have some limited physical content, it is difficult to separate the physical content from the mathematical formalism, and therefore difficult to determine what the basic physical content of the theory is. Given that the extent to which mathematics describes physical phenomena is remarkable, there should nevertheless exist a distinction between the mathematics that quantitatively expresses the laws governing physical phenomena and the physical principles that underlie those laws.

In the traditional developments of the quantum theory, the uncertainty principle is sometimes derived as a consequence of what are made to appear more basic or at least equally basic notions, and the fundamental nature of this principle is therefore obscured. In contrast, it is contended here that the uncertainty principle by itself is sufficient to both justify the entire mathematical formalism underlying the theory and to allow for a derivation of the quantum mechanical equations that result from it. Moreover, it is shown here that the development of the theory based on the uncertainty principle makes evident the fact that the quantum mechanical formalism proves useful only because it allows for a simple quantitative expression of the notion of incompatible observables. It is significant that the few rules and definitions used to define the formalism are no more numerous than those assumed in any formulation of the theory. That the resulting formalism is self consistent and leads to no physical content other than that embedded into it by the notions of uncertainty and invariance is demonstrated in detail in [1].

To make the points above, certain well known equations are restated in Section II and III.A for the purpose of showing that the content of these equations is that contained in Equation (1). Since it is understood that content is added to the theory through the invariance principle, Section III.B is intended to emphasize exactly how its content enters the formalism and to demonstrate that the principle leads to the existence of explicit pairs of conjugate observables. Specifically, it is shown to be a consequence of Equation (1) and the definition of the generator of an infinitesimal displacement, that the observable associated with the generator of the displacement is conjugate to a coordinate observable and has all the properties of the linear momentum ${ }^{1}$.

\footnotetext{
${ }^{1}$ What is emphasized is not the relationship between the generator of a displacement and the linear momentum, which is well known, but that the relationship follows from Equation (1) and the invariance principle.
} 


\section{Development of Formalism}

To construct a quantitative theory to describe physical phenomena it is necessary to make use of some mathematical formalism in terms of which physical principles can be expressed so as to lead to quantitative relations between observables. Because the principle of uncertainty is entirely restrictive in its content, its role is primarily to restrict the mathematical formalism in terms of which the physical theory can be expressed. In principle, the nature of the formalism is arbitrary provided it allows for proper expression of the adopted principles and provided the axioms underlying it are consistent in a rigorous mathematical sense. The well known elements of the quantum formalism are reviewed here only to emphasize that they provide a remarkably simple basis for expression of the single notion that not all observables have exact values at the same time. In a classical theory, the formalism consists of relations between symbols representing the values of observables, and the symbols of the formalism therefore have the properties of mathematical variables. On the other hand, in a quantum theory that takes account of the principle of uncertainty, the formalism of the classical theory must be altered precisely because all observables do not have exact values in a given state of a physical system. Specifically, the formalism must be altered so as to prevent the assignment of precise values to observables that do not have precise values simultaneously. This necessary alteration of the formalism is accomplished most simply by expressing the relations between observables as operator relations in which observables are represented by operators (denoted $O$ ) that act on a symbol called a ket (denoted |\rangle ) which serves to keep track of the accuracy to which values can be assigned to particular observables in a given state. From this point of view the ket can be defined as a listing of the observables that specify a given state, along with the mean values of, and uncertainties in, those observables.

Since the uncertainty principle disallows the possibility that all observables can have precise values simultaneously, it is necessary to distinguish between states in which certain observables have precise values and states in which all observables have only imprecise values. A state in which certain observables have precise values is referred to as an eigenstate of those observables, and the ket that represents it is referred to as an "eigenket". The eigenket represents a listing of the observables and the values of those observables that specify the eigenstate.

In the development of the formalism it is found sufficient to define only one rule of operation for an operator acting on a ket, namely. the rule that determines the result of an operator corresponding to an observable acting on an eigenket of that observable. Given an observable $\alpha$, and an associated operator $O_{\alpha}$ and eigenket $\left|\alpha_{k}\right\rangle$, this one rule of operation is defined by the equation

$$
O_{\alpha}\left|\alpha_{k}\right\rangle=\alpha_{k}\left|\alpha_{k}\right\rangle=\left|\alpha_{k}\right\rangle \alpha_{k},
$$

where $\alpha_{k}$ denotes the eigenvalue of $\alpha$ in the eigenstate represented by $\left|\alpha_{k}\right\rangle$. The sufficiency of this rule follows from the fact that the formulation of the principle of uncertainty results in a prescription whereby any ket is expressible in terms of the eigenkets of any operator relating to the same degrees of freedom; by use of which any operator acting on any ket is expressible in terms of that operator acting on its eigenkets, the result of which is determined by the above rule. More importantly, the above rule has the effect of reducing the quantum formalism to a classical formalism whenever the principle of uncertainty is ignorable. In particular, by use of the above rule, the quantum formalism is such that whenever all operators in an operator equation correspond to observables that can have precise values simultaneously, each operator in the equation can be replaced by a variable representing its eigenvalue; in which case the kets can be canceled from each term and the operator equation can be reduced to a "classical equation".

To connect the formalism to the results of physical measurements it is necessary to connect the elements of the formalism to real numbers. This is done by introducing quantities "conjugate" to kets, known as bras (and denoted \langle| ), and by defining the "expectation value" of an observable. The resulting equations are well known [5]. For every ket $|\Psi\rangle$ a conjugate bra $\langle\Psi|$ is defined such that its product with $|\Psi\rangle$ from the left is a positive real number, denoted by $\langle\Psi \mid \Psi\rangle$. Similarly, for every ket $|\Psi\rangle$ multiplied (on the left) by an operator $O$, a conjugate quantity written $\langle\Psi| \bar{O}$ is defined such that its product with $O|\Psi\rangle$ from the left is a positive real number (denoted $\langle\Psi|\bar{O} O| \Psi\rangle$ ). The operator $\bar{O}$ thus defined is referred to as the "adjoint" of the operator $O$. Finally, for every observable $\alpha$, represented by an operator $O_{\alpha}$, the expectation value of $\alpha$ in the state represented by the ket $|\Psi\rangle$ is defined to equal the quantity

$$
\langle\alpha\rangle=\left\langle\Psi\left|O_{\alpha}\right| \Psi\right\rangle /\langle\Psi \mid \Psi\rangle \text {. }
$$


The rule in Equation (3) is consistent with the basic rule of operation (2) whereby the expectation value of $\alpha$ in an eigenstate of $\alpha$ is reduced to its eigenvalue. To define all potential operations within the formalism it is necessary to include the rule of operation of an operator $O_{\alpha}$ on an "eigenbra" conjugate to an eigenket of the operator. The rule is expressed by the equation $\left\langle\alpha_{k}\right| O_{\alpha}=\left\langle\alpha_{k}\right| \alpha_{k}=\alpha_{k}\left\langle\alpha_{k}\right|$. It then follows that an operator with real eigenvalues must be "self-adjoint" so as to satisfy the equation $\bar{O}=O$. By use of the above equation and Equation (2), it is easy to demonstrate in addition that eigenkets associated with distinct eigenvalues satisfy the familiar "orthogonality relation"

$$
\left\langle\alpha_{k} \mid \alpha_{j}\right\rangle=\delta_{j k}\left\langle\alpha_{j} \mid \alpha_{j}\right\rangle
$$

The definition of the "value" of an observable in Equation (3) makes possible a quantitative definition of the uncertainty in the value of an observable. The uncertainty is defined in analogy with the conventional uncertainty in the result of measurements of an observable $\alpha$, given by the "root-mean-square-deviation" of the measured values from the mean, written $\left.\left[\left\langle\langle\alpha-\langle\langle\alpha\rangle\rangle)^{2}\right\rangle\right\rangle\right]^{1 / 2}$, where the brackets $\langle\langle\rangle\rangle$ denote averages of separate measurements. Because the expectation value plays the role of the average value in the quantum formalism, we define the quantum mechanical uncertainty $\Delta \alpha$ by the equality

$$
\Delta \alpha=\left[\left\langle(\alpha-\langle\alpha\rangle)^{2}\right\rangle\right]^{1 / 2}=\left[\langle\alpha\rangle^{2}-\langle\alpha\rangle^{2}\right]^{1 / 2}
$$

where \langle\rangle denotes the expectation value.

\section{Incorporation of Principles}

\subsection{Uncertainty Principle}

It remains to express the principle of uncertainty quantitatively in the formalism. This is accomplished by defining a "superposition relation" for kets, which results in certain commutation relations for operators. Since the rules of the formalism given above determine only the operation of an operator on an eigenket of that operator, there exists arbitrariness in the formalism relating to the operation of an operator on a ket that is not an eigenket. In particular, it remains to prescribe the operation of an operator $O_{\alpha}$ on a more general ket of the form $\left|\Psi_{k}\right\rangle=\left|[\alpha, \Delta \alpha]_{k}[\beta, \Delta \beta]_{k}\right\rangle$ relating to a state in which two conjugate observables, $\alpha$ and $\beta$, have uncertainties $\Delta \alpha_{k}$ and $\Delta \beta_{k}$. The operation of $O_{\alpha}$ on such a ket needs to be consistent with the basic rule of the formalism which dictates that the operation of $O_{\alpha}$ on an eigenket of $\alpha$ result in the value of $\alpha$ in the state that the ket represents. But since, by its definition, the state represented by the ket has an uncertain value of $\alpha$ in a range $\Delta \alpha_{k}$ about a mean value $\alpha_{k}$, it follows that the operation of $O_{\alpha}$ on the ket cannot result in a single value of $\alpha$, but instead must result in a set of possible values of $\alpha$ within the interval in which $\alpha$ is uncertain.

A prescription for the operation of $O_{\alpha}$ on the above ket consistent with the above requirement can be arrived at by defining the ket to equal a linear combination (or superposition) of the eigenkets of $\alpha$ relating to eigenvalues in the interval $\Delta \alpha_{k}$. The resultant definition is expressed by the equality

$$
\left|\Psi_{k}\right\rangle=\left|[\alpha, \Delta \alpha]_{k}[\beta, \Delta \beta]_{k}\right\rangle=\sum_{\alpha_{j}}^{\Delta \alpha_{k}} C_{j k}(\alpha)\left|\alpha_{j}\right\rangle
$$

(and its counterpoint with $\alpha$ and $\beta$ interchanged). Below, for simplicity, all kets are assumed to be normalized to unity and the eigenvalues $\alpha_{j}$ are taken to be discrete and to lie in the interval between $\alpha_{k}-\Delta \alpha_{k} / 2$ and $\alpha_{k}+\Delta \alpha_{k} / 2$. For generality, the coefficients, $C_{j k}(\alpha)$, of the separate eigenkets in Equation (6) are allowed to be complex numbers (with any dependence on $\beta$ suppressed).It is the superposition relation (6) that assigns kets the properties of "rays" in Hilbert space.

The extent to which superposition relations of the above form provide a proper expression of the principle of uncertainty must depend on the extent to which the equations resulting from the relations can be interpreted in a manner consistent with the principle. In particular, use of the relation (6) in the defining equation (3) must result in an expectation value of $\alpha$ consistent with the uncertainty in $\alpha$. To compute this expectation value in the state represented by the ket $\left|\Psi_{k}\right\rangle$ in Equation (6), a bra conjugate to $\left|\Psi_{k}\right\rangle$ can be constructed in the form 
$\sum_{\alpha_{j}}^{\Delta \alpha_{k}}\left\langle\alpha_{j}\right| C_{k j}^{*}(\alpha)$, where * denotes the complex conjugate. That this bra is conjugate to $\left|\Psi_{k}\right\rangle$ follows from the fact that its product with $\left|\Psi_{k}\right\rangle$ is guaranteed to be a positive real number by the orthogonality of the distinct eigenkets. By use of this bra, combined with Equations (2) and (6) and the orthogonality of the kets $\left|\alpha_{k}\right\rangle$, the expectation value of $\alpha$ in the state represented by $\left|\Psi_{k}\right\rangle$ is evaluated as

$$
\langle\alpha\rangle=\left\langle\Psi_{k}\left|O_{\alpha}\right| \Psi_{k}\right\rangle=\sum_{\alpha_{j}}^{\Delta \alpha} \alpha_{j}\left|C_{j k}(\alpha)\right|^{2}
$$

Here the normalization of all kets to unity guarantees the equality

$$
\sum_{\alpha_{j}}^{\Delta \alpha_{k}}\left|C_{j k}(\alpha)\right|^{2}=1
$$

Equations (7) and (8) show the expectation value of $\alpha$ in the state represented by the ket $\left|\Psi_{k}\right\rangle$ to be a sum over the eigenvalues of $\alpha$ in the interval of uncertainty, with the separate eigenvalues weighted by positive real numbers that sum to unity. The result is exactly that wanted for an average value (!), and has a straightforward interpretation consistent with the uncertainty principle. In particular, because the positive real numbers $\left|C_{j k}(\alpha)\right|^{2}$ sum to unity, they have the characteristic properties that define probabilities, and (in the absence of later inconsistencies) can therefore be identified with the probabilities of the particular values of $\alpha$ within the interval of uncertainty $(\Delta \alpha)_{k}$ in the state $\Psi_{k}$. In the case of continuum eigenvalues and more general normalizations it is necessary only to re-interpret the probabilities as relative probabilities.

The prescription for representation of the ket $\left|\Psi_{k}\right\rangle$ expressed by Equation (6) can therefore be said to be a valid element of the formalism constructed to express the uncertainty principle. Moreover, the appearance of probabilities in the equations of a theory restricted by the uncertainty principle is consistent with the expected probabilistic nature of the theory. On the other hand, it is a consequence of the structure of the formalism that the probabilities enter the formalism as the absolute squares of the coefficients $C_{j k}(\alpha)$ rather than more directly as the coefficients themselves; and from this it follows that the physical content of the theory must be unaffected by a change in the phase of the coefficients $C_{j k}(\alpha)$. The interpretation of the absolute squares of the coefficients combined with the fact that the coefficients themselves are expressible as bra-ket products of the form $C_{j k}(\alpha)=\left\langle\alpha_{j} \mid \Psi_{k}\right\rangle$ (as follows by multiplication of Equation (6) on the left by $\left\langle\alpha_{j}\right|$ ), allows the bra-ket products to be interpreted as probability amplitudes.

Given the form and interpretation of the superposition relation (6), inclusion of terms in the superposition relation with zero coefficients (corresponding to zero probabilities) then allows summations over $\alpha_{j}$ in relations of the form (6) to be extended over the complete set of eigenkets of $\alpha$ associated with all possible eigenvalues of the observable. This makes possible the derivation of a "completeness relation" whereby any ket can be expanded in the eigenkets of any operator that relates to the same degrees of freedom. The existence of a superposition relation with more than one non-zero coefficient then becomes an expression of the uncertainty principle and leads to a non-classical result. The conclusion results from the orthogonality of distinct eigenkets and the fact that, in a classical theory, every ket is an eigenket of every observable, from which it follows, in a classical theory, that if a ket is expanded in eigenkets, all but one of the coefficients must be zero.

A more quantitative expression of the uncertainty principle in the formalism is obtained through an equation for the value of the "commutator" of a pair of operators corresponding to conjugate observables. The equation can be developed from the definition of the uncertainty in an observable $\alpha$ in Equation (5) together with the expression for the expectation value in Equation (3).In particular, by multiplying together the uncertainties in observables $\alpha$ and $\beta$ as expressed by Equation (5), and use of the definition of the expectation value in Equation (3), an expression for the product $\Delta \alpha \Delta \beta$ can be derived in the form [1] [6]

$$
\Delta \alpha \Delta \beta \geq \frac{\left|i\left\langle\left[O_{\alpha}, O_{\beta}\right]\right\rangle\right|}{2}
$$

where the bracket $\left[O_{\alpha}, O_{\beta}\right]$ denotes the commutator, equal to $O_{\alpha} O_{\beta}-O_{\beta} O_{\alpha}$. Combination of this relation with the uncertainty relation in Equation (1) for the case of conjugate observables $\alpha$ and $\beta$ requires the commutator of the operators $O_{\alpha}$ and $O_{\beta}$ to satisfy the relation 


$$
\left[O_{\alpha}, O_{\beta}\right]= \pm i \hbar
$$

consistent with the commutability of operators in the limit of zero $\hbar$. It is important that the relation in Equation (9) allows relation (10) to be derived from the inequality (1) or alternatively allows the inequality (1) to be derived from Equation (10). On the other hand, the commutation relation (10) can be shown to be consistent with the required orthogonality of distinct eigenkets only under certain conditions on the spectrum of eigenvalues $\alpha$ and $\beta$ [7]. Specifically, in the case where the eigenvalues of $\alpha$ and $\beta$ lie in a continuum, it is required that the ranges of the eigenvalues extend between plus and minus infinity. In this case, the orthogonality and completeness relations for the eigenkets of $\alpha$ and $\beta$ assume the forms [5]

$$
\begin{aligned}
& \left\langle\alpha \mid \alpha^{\prime}\right\rangle=\delta\left(\alpha-\alpha^{\prime}\right),\left\langle\beta \mid \beta^{\prime}\right\rangle=\delta\left(\beta-\beta^{\prime}\right) \\
& \int \mathrm{d} \alpha|\alpha\rangle\left\langle\alpha\left|=1, \int \mathrm{d} \beta\right| \beta\right\rangle\langle\beta|=1 .
\end{aligned}
$$

The relations (11) combined with Equation (2) make possible a re-expression of the operator Equation (10) as an ordinary equation connecting functions of $\alpha$ and $\beta$. The result is obtained by multiplication of Equation (10) on the left and right respectively by eigenbras and eigenkets of $\alpha$ to produce the relation

$$
\left\langle\alpha^{\prime}\left|\left[O_{\alpha}, O_{\beta}\right]\right| \alpha^{\prime \prime}\right\rangle= \pm i \hbar\left\langle\alpha^{\prime} \mid \alpha^{\prime \prime}\right\rangle
$$

which Equations (2) and (11) (and choice of the "+" sign) reduce to the form

$$
\left(\alpha^{\prime}-\alpha^{\prime \prime}\right)\left\langle\alpha^{\prime}\left|O_{\beta}\right| \alpha^{\prime \prime}\right\rangle=i \hbar \delta\left(\alpha^{\prime}-\alpha^{\prime \prime}\right) \text {. }
$$

The extremely singular character of this last relation (for $\alpha^{\prime}=\alpha^{\prime \prime}$ ), in comparison with the delta function relation

$$
\alpha \frac{\mathrm{d}}{\mathrm{d} \alpha} \delta(\alpha)=-\delta(\alpha)
$$

then requires the quantity $\left\langle\alpha^{\prime}\left|O_{\beta}\right| \alpha^{\prime \prime}\right\rangle$ to be proportional to the derivative of a delta-function. Specifically, comparison between Equations (12) and (13) leads to the equivalence

$$
\left\langle\alpha^{\prime}\left|O_{\beta}\right| \alpha^{\prime \prime}\right\rangle=-i \hbar \frac{\partial}{\partial \alpha^{\prime}} \delta\left(\alpha^{\prime}-\alpha^{\prime \prime}\right)=-i \hbar \frac{\partial}{\partial \alpha^{\prime}}\left\langle\alpha^{\prime} \mid \alpha^{\prime \prime}\right\rangle .
$$

The equivalence can be reworked into a more general relation (and its adjoint) by expansion of an arbitrary ket $|\Psi\rangle$ (relating to the degrees of freedom of the observables $\alpha$ and $\beta$ ) in terms of eigenkets of $\alpha$ in the form

$$
|\Psi\rangle=\int \mathrm{d} \alpha^{\prime \prime}\left|\alpha^{\prime \prime}\right\rangle\left\langle\alpha^{\prime \prime} \mid \Psi\right\rangle
$$

The expansion makes it possible to rewrite Equation (14) as the more general relation

$$
\left\langle\alpha^{\prime}\left|O_{\beta}\right| \Psi\right\rangle=-i \hbar \frac{\partial}{\partial \alpha^{\prime}}\left\langle\alpha^{\prime} \mid \Psi\right\rangle
$$

which (combined with the symmetry between $\alpha$ and $\beta$ ) serves to establish the well known prescriptions that define the result of an observable operator acting on a continuum eigenket or eigenbra of a conjugate observable

$$
\begin{aligned}
\left\langle\alpha^{\prime}\right| O_{\beta} & =-i \hbar \frac{\partial}{\partial \alpha^{\prime}}\left\langle\alpha^{\prime}\left|, \quad O_{\beta}\right| \alpha^{\prime \prime}\right\rangle=i \hbar \frac{\partial}{\partial \alpha^{\prime \prime}}\left|\alpha^{\prime \prime}\right\rangle, \\
\left\langle\beta^{\prime}\right| O_{\alpha} & =i \hbar \frac{\partial}{\partial \beta^{\prime}}\left\langle\beta^{\prime}\left|, \quad O_{\alpha}\right| \beta^{\prime \prime}\right\rangle=-i \hbar \frac{\partial}{\partial \beta^{\prime \prime}}\left|\beta^{\prime \prime}\right\rangle,
\end{aligned}
$$

with the lack of complete symmetry between $\alpha$ and $\beta$ a reflection of the particular choice of sign in the commutation relation of Equation (10).

The prescriptions (17), together with the rule (2), lead immediately to a differential equation for the bra-ket product $\left\langle\alpha^{\prime} \mid \beta^{\prime}\right\rangle$ in the form

$$
\left\langle\alpha^{\prime}\left|O_{\beta}\right| \beta^{\prime}\right\rangle=-i \hbar \frac{\partial}{\partial \alpha^{\prime}}\left\langle\alpha^{\prime} \mid \beta^{\prime}\right\rangle=\beta^{\prime}\left\langle\alpha^{\prime} \mid \beta^{\prime}\right\rangle
$$


which has the solution

$$
\left\langle\alpha^{\prime} \mid \beta^{\prime}\right\rangle=\left\langle\beta^{\prime} \mid \alpha^{\prime}\right\rangle^{*}=C \mathrm{e}^{\frac{i \alpha^{\prime} \beta^{\prime}}{\hbar}},
$$

where $C$ is an arbitrary complex constant, independent of both $\alpha^{\prime}$ and $\beta^{\prime}$. Equation (19) shows the bra-ket product $\left\langle\alpha^{\prime} \mid \beta^{\prime}\right\rangle$ (symmetric in $\alpha^{\prime}$ and $\beta^{\prime}$ ) to be a periodic function of the eigenvalues of the conjugate observables $\alpha$ and $\beta$, with a period in $\alpha$ given by $2 \pi \hbar / \beta^{\prime}=h / \beta$ and a period in $\beta$ given by $2 \pi \hbar / \alpha^{\prime}$. The establishment of the uncertainty relation $\Delta x \Delta p_{x} \geq \hbar / 2$ below, combined with the simple analysis leading to Equation (19), is then sufficient to establish that the probability amplitude $\left\langle x \mid p_{x}\right\rangle$ is a periodic function of $x$ with a (de Broglie) wavelength in space given by $h / p_{x}$. More importantly for the point to be made here, Equations (18) and (19) show that the content of Equations (17) is only that contained in the uncertainty relation (1). In particular, since the bra-ket product determined by Equation (19) is a pure phase factor, the absolute square of which is independent of both $\alpha^{\prime}$ and $\beta^{\prime}$, it is a consequence of the interpretation of $\left|\left\langle\alpha^{\prime} \mid \beta^{\prime}\right\rangle\right|^{2}$ as the (relative) probability that the observable $\alpha$ has the specific value $\alpha^{\prime}$ in the eigenstate of the conjugate observable $\beta$, specified by the value $\beta^{\prime}$, that in an eigenstate specified by a value of $\beta$, all values of the conjugate observable $\alpha$ are equally likely (and vice-versa with $\alpha$ and $\beta$ interchanged). It is therefore the content of Equation (18) that, when the value of $\beta$ is known, the value of $\alpha$ is totally unknown (and vice-versa), consistent with the uncertainty relation in Equation (1), which requires that in an eigenstate defined by a zero uncertainty in the eigenvalue of one member of a pair of conjugate observables, the uncertainty in the eigenvalue of the other member of the pair is infinite. It can be concluded that the four equations (1), (10), (17), and (19) contain only the limited content that the product of the uncertainties in two conjugate observables must equal or exceed $\hbar$, as a consequence of which, whenever one of the observables has an exact value, all values of the other observable are equally likely. The formalism defined above therefore correctly serves the purpose for which it was designed. It expresses the content of Equation (1).

The physical content of the formalism is increased by the addition of the principle of invariance. As an example of this, in the section below, the results obtained from this principle are derived in the simple case of invariance of the equations of the theory with respect to space translations. The results extracted from the invariance principle applied to the complete set of transformations in the Lorentz group are detailed in Reference [1].

\subsection{Invariance Principle}

The recognition of the existence of equivalent space-time frames relative to which the equations of physics should have the same form represents the essential notion underlying the "physical principle of invariance". The content of the principle is then contained in the assertions that define certain distinct frames to be equivalent. Here, for simplicity, we focus only on the asserted physical equivalence of coordinate frames connected to one another by either a shift in the coordinate origin or a rotation of the coordinate axes. In either case, the space coordinates of a system with respect to the initial frame will be labeled $x, y, z$, while the coordinates of the same system with respect to the "transformed frame" will be labeled $x^{\prime}, y^{\prime}, z^{\prime}$. The principle of invariance of course represents a basic physical principle in a classical theory as well as in quantum theory. But the combination of the principle with the uncertainty principle in the quantum theory leads to consequences that have no direct correspondence in classical theory. To combine the two principles in the quantum theory it is necessary to formulate the invariance principle in the formalism constructed to express the uncertainty principle. This is accomplished by the introduction into the quantum theory formalism of transformation operators defined to determine the effects of coordinate transformations on the kets, bras, and operators of the theory. Specifically, since the observables of a system in a given state, in general, have different values when measured with respect to different coordinate frames, the expectation values of operators $O_{\alpha}$ computed for a given state $\Psi$ must in general be different in different frames. This requires that, either the bras and kets change under the coordinate transformation, or the operators change under the transformation. The first choice is referred to here as the "ket picture", and the second as the "operator picture".

In the ket picture, a transformation between an unprimed and a primed coordinate frame is asserted to leave the operators unaltered,

$$
O_{\alpha} \rightarrow O_{\alpha}^{\prime}=O_{\alpha}
$$

but is defined to result in a change in the bras and kets that describe the system by way of the prescriptions 


$$
|\Psi\rangle \rightarrow\left|\Psi^{\prime}\right\rangle=U|\Psi\rangle,\langle\Psi| \rightarrow\left\langle\Psi^{\prime}\right|=\langle\Psi| \bar{U}
$$

with the quantity $U$ defined as the "transformation operator" and $\bar{U}$ defined to be its adjoint. In this picture, the expectation values of physical observables transform according to the relation

$$
\langle\alpha\rangle=\left\langle\Psi\left|O_{\alpha}\right| \Psi\right\rangle \rightarrow\left\langle\alpha^{\prime}\right\rangle=\left\langle\Psi^{\prime}\left|O_{\alpha}\right| \Psi^{\prime}\right\rangle=\left\langle\Psi\left|\bar{U} O_{\alpha} U\right| \Psi\right\rangle
$$

where the change in the expectation value $\langle\alpha\rangle$ results from the changes in $|\Psi\rangle$ and $\langle\Psi|$. Alternatively, in the operator picture, a transformation between primed and unprimed frames is defined to leave the bras and kets unaltered so that

$$
|\Psi\rangle \rightarrow\left|\Psi^{\prime}\right\rangle=|\Psi\rangle,\langle\Psi| \rightarrow\left\langle\Psi^{\prime}\right|=\langle\Psi|
$$

but to result in a change in the operators according to the relation

$$
O_{\alpha} \rightarrow O_{\alpha}^{\prime} \text {. }
$$

In this case the expectation values of physical observables transform as

$$
\langle\alpha\rangle=\left\langle\Psi\left|O_{\alpha}\right| \Psi\right\rangle \rightarrow\left\langle\alpha^{\prime}\right\rangle=\left\langle\Psi\left|O_{\alpha}^{\prime}\right| \Psi\right\rangle
$$

where the change in $\langle\alpha\rangle$ results from the change in $O_{\alpha}$. But because the change in the value of the observable needs to be independent of the choice of the "picture", the two transformation Equations (22a) and (22b) must give the same result; and this requires that the operators $O_{\alpha}$ and $O_{\alpha}^{\prime}$ be connected by the equation

$$
O_{\alpha}^{\prime}=\bar{U} O_{\alpha} U \text {. }
$$

Additional physical content is introduced into the definition of the transformation operators by the requirement of conservation of probability below. In the case of a linear transformation, this requirement restricts the transformation operator $U$ to be unitary, as defined by the equation (see below),

$$
\bar{U}=U^{-1},
$$

in which case Equation (25) can be rewritten in the form $O_{\alpha}^{\prime}=U^{-1} O_{\alpha} U$. The definitions serve to connect every coordinate transformation between physically equivalent frames to a unitary operator $U$ defined to effect the change induced by the transformation in the bras, kets, and operators of the formalism. But, in general, the unitary operator $U$ can in turn be connected to a self-adjoint operator, with real eigenvalues that can be associated with an observable; and this connection allows every coordinate transformation to be connected to a dynamical quantity that serves to define the state of the system considered.

It is sufficient here to consider the case of continuous transformations that can be evolved from the identity operator I by a continuous sequence of transformations generated by infinitesimal variations in a parameter a. In this case, the transformation operator $U$ can in general be represented as

$$
U=I+\delta U=I+i G \delta a,
$$

where $\delta a$ denotes the increment in the transformation parameter, and $d U \equiv i G \delta a$ denotes the corresponding shift in the transformation operator from the identity operator. The operator $G$ defined by the last equation is referred to as the "generator of the infinitesimal transformation". As a consequence of the unitarity of $U$ and introduction of the imaginary $i$ into the definition of $G, G$ must be self-adjoint,

$$
\bar{U} U=I \rightarrow \bar{G}=G \text {. }
$$

We focus here on a transformation between the physically equivalent frames of a closed system resulting from an infinitesimal translation of the coordinate origin by an amount $\delta a_{k}$ (in the negative direction) along the coordinate axis $x_{k}$. The transformation is defined by a connection between the coordinates of a space point in the primed and unprimed frames expressed by the equation

$$
x_{k}^{\prime}=x_{k}+\delta a_{k},
$$

where $\delta a_{k}$ can be identified with the increment in the transformation parameter. In this case the transformation operator $U$ assumes the form

$$
U\left(\delta a_{k}\right)=I+i G_{k} \delta a_{k} .
$$




\subsubsection{Ket Picture of Translation}

Consider first the ket picture of the coordinate transformation in which the operator $U\left(\delta a_{k}\right)$ is defined by the equation

$$
\left|\Psi^{\prime}\right\rangle=U\left(\delta a_{k}\right)|\Psi\rangle
$$

where the kets $\left|\Psi^{\prime}\right\rangle$ and $|\Psi\rangle$ represent a given state of the given system as observed in the primed and unprimed coordinate frames respectively. It is useful to focus initially on a simple system whose translational motion with respect to the $\mathrm{k}$-th coordinate axis is describable in terms of a single space coordinate $x_{k}$ that can be taken to represent the k-th coordinate of the system's "center of mass". In this case the complete set of eigenkets of the observable $x_{k}$ forms a basis for a representation of the kets of the system with respect to the translational degree of freedom along the k-th axis; and a representation of the kets $\left|\Psi^{\prime}\right\rangle$ and $|\Psi\rangle$ with respect to this degree of freedom can be constructed in the primed and unprimed frames from the sets of representatives $\left\langle x_{k}^{\prime} \mid \Psi^{\prime}\right\rangle$ and $\left\langle x_{k} \mid \Psi\right\rangle$ respectively. The content of the invariance principle enters the formalism through the requirement that the probability for the system to have a coordinate corresponding to a given physical point have the same value as measured in the equivalent frames. The requirement is guaranteed only under the condition that the magnitudes of the coordinate representatives of the kets $\left\langle x_{k} \mid \Psi\right\rangle$ and $\left\langle x_{k}^{\prime} \mid \Psi^{\prime}\right\rangle$, relating to the given physical point as measured in the two frames, be equal. This condition requires that the transformation operator $U$ be unitary by way of the equation

$$
\left\langle x_{k}^{\prime} \mid \Psi^{\prime}\right\rangle=\left\langle x_{k}\left|\bar{U}\left(\delta a_{k}\right) U\left(\delta a_{k}\right)\right| \Psi\right\rangle=\left\langle x_{k} \mid \Psi\right\rangle .
$$

The result (32) combined with Equations (29), (30), and (31) is sufficient to determine the properties of the operator $G$, and in addition demonstrate that the observable associated with the related operator $-\hbar G_{k}$ is conjugate to the coordinate observable $x_{k}$.

The derivation proceeds on the basis of well known steps. By use of Equations (29), (30), and (31) the primed frame quantities $x_{k}^{\prime}$ and $\left|\Psi^{\prime}\right\rangle$ on the left hand side of Equation (32) can be re-expressed in terms of their unprimed frame equivalents and Equation (32) can be rewritten in the form

$$
\left\langle x_{k}^{\prime} \mid \Psi^{\prime}\right\rangle=\left\langle x_{k}+\delta a_{k}\left|U\left(\delta a_{k}\right)\right| \Psi\right\rangle=\left\langle x_{k}+\delta a_{k} \mid \Psi\right\rangle+i \delta a_{k}\left\langle x_{k}+\delta a_{k}\left|G_{k}\right| \Psi\right\rangle=\left\langle x_{k} \mid \Psi\right\rangle .
$$

The functions of $x_{k}$ evaluated at $x_{k}+\delta a_{k}$ in this last equation can then be re-expressed in terms of functions evaluated at $x_{k}$ by Taylor series expansions of the functions about $x_{k}$; which, since $\delta a_{k}$ represents an infinitesimal, can accurately be represented by the first two terms in each series. In particular the quantities $\left\langle x_{k}+\delta a_{k} \mid \Psi\right\rangle$ and $\left\langle x_{k}+\delta a_{k}\left|G_{k}\right| \Psi\right\rangle$ can be expressed by the forms

$$
\begin{aligned}
& \left\langle x_{k}+\delta a_{k} \mid \Psi\right\rangle=\left\langle x_{k} \mid \Psi\right\rangle+\delta a_{k} \frac{\partial}{\partial x_{k}}\left\langle x_{k} \mid \Psi\right\rangle, \\
& \left\langle x_{k}+\delta a_{k}\left|G_{k}\right| \Psi\right\rangle=\left\langle x_{k}\left|G_{k}\right| \Psi\right\rangle+\delta a_{k} \frac{\partial}{\partial x_{k}}\left\langle x_{k}\left|G_{k}\right| \Psi\right\rangle .
\end{aligned}
$$

By substitution of Equations (34) into Equation (33) and the neglect of terms proportional to the square of $\delta a_{k}$ one obtains the equation

$$
\left\langle x_{k} \mid \Psi\right\rangle+\delta a_{k} \frac{\partial}{\partial x_{k}}\left\langle x_{k} \mid \Psi\right\rangle+i \delta a_{k}\left\langle x_{k}\left|G_{k}\right| \Psi\right\rangle=\left\langle x_{k} \mid \Psi\right\rangle
$$

which reduces to the result

$$
\left\langle x_{k}\left|G_{k}\right| \Psi\right\rangle=i \frac{\partial}{\partial x_{k}}\left\langle x_{k} \mid \Psi\right\rangle .
$$

The result is sufficient to establish that the observable $-\hbar G_{k}$ has the defining property of an observable conjugate to the coordinate observable $x_{k}$. Specifically, with the operator $-\hbar G_{k}$ denoted by $O_{P k}$,

$$
O_{P k} \equiv-\hbar G_{k},
$$

the form of Equation (36), in terms of the operator $O_{P k}$, is identical to the form of the general Equation (16) which prescribes the result of an operator $O_{\beta}$ corresponding to an observable $\beta$ acting on an eigenbra of a conjugate 
observable $\alpha$. That is

$$
\left\langle x_{k}\left|O_{P k}\right| \Psi\right\rangle=-i \hbar \frac{\partial}{\partial x_{k}}\left\langle x_{k} \mid \Psi\right\rangle .
$$

But, since Equation (16) derives from the commutation relation (10) that represents an expression of the uncertainty relation $\Delta \alpha \Delta \beta \geq \hbar / 2$, it can be expected to follow from Equation (36)' that the operator $O_{P k}$ satisfies the commutation relation

$$
\left[O_{x_{k}}, O_{P_{k}}\right]=i \hbar
$$

which in turn implies that the observable $P_{k}$, associated with the self-adjoint operator $O_{P k}$, satisfies the uncertainty relation

$$
\Delta x_{k} \Delta P_{k} \geq \frac{\hbar}{2}
$$

That the operator $O_{P k}$ does satisfy the commutation relation (38) can be shown to follow from the definition of the two operators $O_{P k}$ and $U\left(\delta a_{k}\right)$ in the operator picture of the coordinate transformation. It is possible to first generalize the result obtained above to the case of a system that can be resolved into an arbitrary number of subsystems labeled n, each of which can be localized in space by the specification of a single coordinate vector $\boldsymbol{x}_{(n)}$ [1]. The generalization results in equations that demonstrate that the operator $O_{P k}$ can be interpreted as the k-th component of a vector operator $O_{P}$, decomposable into a set of operators that separately relate to the independent coordinate vectors required to describe the translational degrees of freedom of the total system. The vector observable $\boldsymbol{P}$ associated with the operator $O_{\boldsymbol{P}}$ can be shown to have the properties corresponding to the total linear momentum observable of classical mechanics [1].

\subsubsection{Operator Picture of Translation}

Consider now the operator picture of the infinitesimal translation in which an operator $O_{\alpha}^{\prime}$ associated with an observable $\alpha$ in the primed frame is connected via the transformation operator $U\left(\delta a_{k}\right)$ to the corresponding operator in the unprimed frame by the equation

$$
O_{\alpha}^{\prime}=\bar{U}\left(\delta a_{k}\right) O_{\alpha} U\left(\delta a_{k}\right) .
$$

By use of Equations (30) and (37) and the self-adjointness of the operator $O_{P k}$, this equation can be re-expressed to terms of order $\delta a_{k}$ in the form

$$
O_{\alpha}^{\prime}=\left(I+\frac{i}{\hbar} \delta a_{k} O_{P k}\right) O_{\alpha}\left(I-\frac{i}{\hbar} \delta a_{k} O_{P k}\right)=O_{\alpha}+\frac{i}{\hbar} \delta a_{k}\left[O_{P k}, O_{\alpha}\right] .
$$

The interest is in the connection between the operators corresponding to coordinate observables $\boldsymbol{x}_{(n)}$ in the primed and unprimed frames. In the general case of a system that can be resolved into an arbitrary number of sub-systems $(1), \cdots,(n), \cdots$, which can each be localized in space by the specification of a single coordinate vector, the k-th components of the complete set of coordinate vectors $\boldsymbol{x}_{(n)}$ in the primed and unprimed frames can be used to describe the translational motion of the total system with respect to the k-th axis; and the complete set of eigenkets of these observables in the two frames can then be used as a basis for a coordinate representation of the kets of the system. In the operator picture the eigenkets of the coordinate observables,

$\left|x_{(1)_{k}}, x_{(2)_{k}}, \cdots, x_{(n)_{k}}, \cdots\right\rangle$ are defined to be the same in both the primed and unprimed frames, while the eigenvalues of the coordinate operators are required to be related by Equation (29). It follows that the coordinate operators in the primed and unprimed frames, $O_{x_{(n)_{k}}}^{\prime}$ and $O_{x_{(n)_{k}}}$, must satisfy the equations

$$
\begin{aligned}
O_{x_{(n)_{k}}}\left|x_{(1)_{k}}, \cdots, x_{(n)_{k}}, \cdots\right\rangle & =x_{(n)_{k}}\left|x_{(1)_{k}}, \cdots, x_{(n)_{k}}, \cdots\right\rangle, \\
O_{x_{(n)_{k}}}^{\prime}\left|x_{(1)_{k}}, \cdots, x_{(n)_{k}}, \cdots\right\rangle & =x_{(n)_{k}}^{\prime}\left|x_{(1)_{k}}, \cdots, x_{(n)_{k}}, \cdots\right\rangle=\left(x_{(n)_{k}}+\delta a_{k}\right)\left|x_{(1)_{k}}, \cdots, x_{(n)_{k}}, \cdots\right\rangle .
\end{aligned}
$$

from which it follows that the operator $O_{x_{(n)_{k}}}^{\prime}$ acting on the eigenket $\left|x_{(1)_{k}}, \cdots, x_{(n)_{k}}, \cdots\right\rangle$ is equivalent to the 
operator $\left(O_{x_{(n)_{k}}}+\delta a_{k} I\right)$ acting on the same eigenket. The equivalence can be formulated as an operator equation, which is valid when the operators in the equation act on any ket:

$$
O_{x_{(n)_{k}}}^{\prime}=O_{x_{(n)_{k}}}+\delta a_{k} I .
$$

In particular, since the above connection between the operators $O_{x_{(n)_{k}}}^{\prime}$ and $O_{x_{(n)_{k}}}$ is valid when the operators act on the eigenkets $\left|x_{(1)_{k}}, \cdots, x_{(n)_{k}}, \cdots\right\rangle$, and since any ket relating to the given system's translational degrees of freedom along the $\mathrm{k}$-th axis can be expanded in terms of the eigenkets $\left|x_{(1)_{k}}, \cdots, x_{(n)_{k}}, \cdots\right\rangle$, the connection between the operators must be valid when the operators act on any ket on which they can act. But as a consequence of Equation (41), the operators $O_{x_{(n)_{k}}}^{\prime}$ and $O_{x_{(n)_{k}}}$ are connected by the equation

$$
O_{x_{(n)_{k}}}^{\prime}=O_{x_{(n)_{k}}}+\frac{i}{\hbar} \delta a_{k}\left[O_{P_{k}}, O_{x_{(n)_{k}}}\right]
$$

and it follows from comparison of Equations (44) and (45) that the operators $O_{x_{(n)_{k}}}$ and $O_{P_{k}}$ need to satisfy the commutation relation

$$
\left[O_{x_{(n)_{k}}}, O_{P_{k}}\right]=i \hbar
$$

Here the operator $O_{P_{k}}$ can be interpreted as the k-th component of a vector operator, which itself can be decomposed into a set of operators $O_{P(n)_{k}}$ that separately relate to the independent coordinate vectors required to describe the translational degrees of freedom of the total system. Specifically, the operator $O_{P_{k}}$ can be represented by the sum

$$
O_{P_{k}}=\sum_{n} O_{P(n)_{k}}
$$

by use of which Equation (46) can be reduced to an equality relating strictly to the degrees of freedom connected to the coordinate vector $\boldsymbol{x}_{(n)}$. In particular, as a consequence of the fact that operators relating to distinct degrees of freedom must commute, the decomposition of the operator $O_{P_{k}}$ given by Equation (47) allows Equation (46) to be re-expressed as the relation

$$
\left[O_{x_{(n)}}, O_{P(r)_{j}}\right]=i \hbar \delta_{(n)(r)} \delta_{j k} .
$$

It then follows from the fact that the derived commutation relation between the operators $O_{x_{(n)_{k}}}$ and $O_{P(r)_{j}}$ is identical to the commutation relation between operators associated with conjugate observables, that the observables $x_{(n)_{k}}$ and $p_{(n)_{k}}$ must satisfy the uncertainty relation

$$
\Delta x_{(n)_{k}} \Delta p_{(n)_{k}} \geq \frac{\hbar}{2} .
$$

The conclusion extracted from the operator picture of the coordinate translation is therefore the same as that deduced from the ket picture of the transformation (as it should be). In fact, because Equation (36)' can be derived from Equation (46), the property of the operator $O_{P_{k}}$ expressed in Equation (46) in the operator picture is exactly equivalent to the property of $O_{P_{k}}$ expressed in Equation (36)' in the ket picture, consistent with the required arbitrariness in the choice of either picture; and the content of Equation (48) is only that in Equation (49).

Similarly, by considering the connections between coordinate frames related by a rotation of the coordinate axes rather than by a translation of the origin, an analysis parallel to the above demonstrates that the observable associated with the (properly defined) generator of an infinitesimal rotation about the k-th coordinate axis, $O_{L_{k}}$, is conjugate to the azimuthal angle coordinate $\varphi_{k}$ in the plane perpendicular to the k-th axis [7]. The result can be expressed in the form of the uncertainty relation

$$
\Delta \varphi_{k} \Delta L_{k} \geq \frac{\hbar}{2}
$$

with the operator associated with the observable $L_{k}$ connected to the generator of the infinitesimal rotation, $G_{k}$, by way of the equation 


$$
O_{L_{k}} \equiv \hbar G_{k}
$$

It can be demonstrated that the observables associated with the generators of infinitesimal translations and rotations have in fact all the familiar properties of linear and angular momentum observables [1]. Specifically, the invariance principle requirement that the equations of motion of a closed system be unaltered by a translation or rotation demands that the generators of the transformations commute with the Hamiltonian and that their eigenvalues therefore be constants of the motion. In addition, it is shown below that the linear momentum observable is connected in the usual way to the energy observable, which itself can be associated with the generator of an infinitesimal translation of the origin of time. The overall conservation of linear momentum and energy can then be derived from the invariance principle requirement that the equations of motion of a closed system be invariant in form under translations of the origins of the space and time axes. The connection between the energy observable and the generator of a time translation also allows for a rigorous derivation of an energy-time uncertainty relation expressing the well known connection between the energy "width" and the lifetime of a state ${ }^{2}$ [1] [8].

It is important that Equation (36)' in the form

$$
-i \hbar \frac{\partial}{\partial x_{k}}\left\langle\boldsymbol{x}_{(\mathrm{cm})} \mid \Psi\right\rangle=\left\langle\boldsymbol{x}_{(\mathrm{cm})}\left|O_{\boldsymbol{P}_{k}}\right| \Psi\right\rangle,
$$

where $\boldsymbol{x}_{(\mathrm{cm})}$ denotes the center of mass coordinate, connects the operator $O_{\boldsymbol{P}_{k}}$ to a space derivative in the same way that the definition of the Hamiltonian $H$ as the generator of a time shift connects $H$ to a time derivative through the equation

$$
i \hbar \frac{\mathrm{d}}{\mathrm{d} t}\left\langle\boldsymbol{x}_{(\mathrm{cm})} \mid \Psi\right\rangle=\left\langle\boldsymbol{x}_{(\mathrm{cm})}|H| \Psi\right\rangle .
$$

The covariance of the equations under the complete set of Lorentz transformations requires that the two equations be combinable into a four-vector equation. This can be guaranteed only under the condition that the operators $O_{P}$ and $H / c$ can be defined to be the space and time components of a single fourvector $O_{P_{\mu}}(\mu=0,1,2,3)$ (with $\mathrm{c}$ the speed of light). In the case of a closed system, for which $c O_{P}$ and $H$ much commute, the simultaneous eigenvalues of the two operators, $c \boldsymbol{P}$, and $E$ form a four-vector, the "scalar" product with itself of which must have an invariant (constant) value in physically equivalent frames, as expressed by the equation

$$
E^{2}-(c \boldsymbol{P})^{2}=\text { Constant } \equiv \kappa^{2} .
$$

But when an equation involves only simultaneous observables, the equation must be valid when the values of the observables are replaced by their corresponding operators acting on any ket $|\Psi(t)\rangle$ that can be expanded in terms of the eigenkets of the operators. This allows Equation (54) to be re-expressed as the operator equation

$$
\left(H^{2}-c^{2} O_{P}^{2}\right)|\Psi(t)\rangle=\kappa^{2}|\Psi(t)\rangle .
$$

Multiplication of Equation (55) on the left by a coordinate eigenbra $\left\langle\boldsymbol{x}_{(\mathrm{cm})}\right|$ and use of Equations (52) and (53) (twice) to replace the operators by derivatives converts this equation into the (free particle) Klein-Gordon equation expressible as

which the notation

$$
\left[\frac{1}{c^{2}} \frac{\partial^{2}}{\partial t^{2}}-\nabla^{2}+\frac{\kappa^{2}}{(\hbar c)^{2}}\right]\left\langle\boldsymbol{x}_{(\mathrm{cm})} \mid \Psi(t)\right\rangle=0,
$$

$$
\boldsymbol{x}_{(\mathrm{cm})}=\boldsymbol{x},\left\langle\boldsymbol{x}_{(\mathrm{cm})} \mid \Psi(t)\right\rangle=\Psi(\boldsymbol{x}, t), m=\frac{\kappa^{2}}{c^{2}}
$$

re-expresses as

$$
\left[\frac{1}{c^{2}} \frac{\partial^{2}}{\partial t^{2}}-\nabla^{2}+\left(\frac{m c}{\hbar}\right)^{2}\right] \Psi(\boldsymbol{x}, t)=0 .
$$

\footnotetext{
${ }^{2}$ Because time needs to be interpreted as a parameter in the theory rather than an observable, the derivation and interpretation of the uncertainty relation connecting energy and time are very different from those developed here for the observables $x$ and $p_{x}$.
} 
An approximate equation for a free particle can be derived from this equation by solution of Equation (54) for the energy eigenvalue $E$ in terms of a square root, and approximation of the square root in the case in which $c p \ll \kappa$. The approximation reduces Equation (58) to the free particle Schrodinger equation

$$
i \hbar \frac{\partial}{\partial t} \Psi(\boldsymbol{x}, t)=\left(-\frac{\hbar^{2}}{2 m} \nabla^{2}+m c^{2}\right) \Psi(\boldsymbol{x}, t)=0 .
$$

The validity of Equations (58) and (59) is limited by the fact that the forms of the equations are altered by a phase change in the function $\Psi(\boldsymbol{x}, t)$. Instead, where $\Lambda(\boldsymbol{x}, t)$ is an arbitrary real phase function, $\Psi(\boldsymbol{x}, t)$ and $\Psi(x, t) \mathrm{e}^{i \Lambda(x, t)}$ can satisfy the same equations only if Equations (58) and (59) are modified by the inclusion of additional terms that can compensate for the derivatives of the phase function $\Lambda(\boldsymbol{x}, t)$ in the solution of the equations. The added terms can do this only provided they add directly to the derivative operators in the equation, and their physical significance is unaltered by the addition of derivatives of an arbitrary function of $x$ and $t$. Specifically, it can be shown [1] that the phase invariance of an equation with the derivative structure of Equation (58) is guaranteed only if the equation is modified by replacement of the space and time derivatives according to the prescriptions

$$
\frac{\partial}{\partial x_{k}} \rightarrow \frac{\partial}{\partial x_{k}}-i A_{k}(\boldsymbol{x}, t), \frac{\partial}{\partial t} \rightarrow \frac{\partial}{\partial t}+i A_{0}(\boldsymbol{x}, t),
$$

where $A_{k}$ and $A_{0}$ are the space and time components of a four-vector function of $x$ and $t$, and where values of physical observables derived from the functions $A_{k}$ and $A_{0}$ are unchanged by the following transformations

$$
\begin{aligned}
& A_{0}(\boldsymbol{x}, t) \rightarrow A_{0}^{\prime}(\boldsymbol{x}, t)=A_{0}(\boldsymbol{x}, t)-\frac{\partial \Lambda(\boldsymbol{x}, t)}{\partial t} \\
& A_{k}(\boldsymbol{x}, t) \rightarrow A_{k}^{\prime}(\boldsymbol{x}, t)=A_{k}(\boldsymbol{x}, t)+\frac{\partial \Lambda(\boldsymbol{x}, t)}{\partial x_{k}}
\end{aligned}
$$

with $\Lambda(\boldsymbol{x}, t)$ an arbitrary function of $x$ and $t$. After omission of a non-dynamical "rest energy term", $m c^{2}$, and with $A_{k}(k=1,2,3)$ set to zero, the prescriptions (60) convert Equation (59) into the non-relativistic "Schrodinger equation",

$$
i \hbar \frac{\partial}{\partial t} \Psi(\boldsymbol{x}, t)=\left(-\frac{\hbar^{2}}{2 m} \nabla^{2}+\bigvee(\boldsymbol{x}, t)\right) \Psi(\boldsymbol{x}, t),
$$

with $\mathrm{V}=\hbar c A_{0}$. But because the addition of space and time dependent functions to the basic equations of the theory is inconsistent with the invariance of the equations under space and time translations, the transformed equations cannot describe a closed system, and therefore cannot describe a free particle. Instead, the added terms can be associated with an influence on the described system dependent on the system's location in space and time, which influence can only be interpreted in terms of an interaction of the system with an external system. From this it follows that the equation of motion of a particle can satisfy the requirement of local phase invariance only if the particle can couple to another system through an interaction. Moreover, the phase invariance condition serves to determine the way in which the interaction terms can be introduced into the basic equations of the theory.

\section{Summary and Conclusion}

What is demonstrated here is that combination of the invariance principle with only a weak statement of the uncertainty principle that asserts only the existence of unspecified pairs of conjugate observables is sufficient to establish the existence of specific pairs of such observables, which in fact exhaust the complete set of known pairs of observables satisfying uncertainty relations of the form (1). It is shown in addition that the invariance principle, formulated within the quantum formalism, determines the forms of the basic "equations of motion" of the theory.

\section{Acknowledgements}

The author thanks Professor D. G. Ellis for useful comments relating to this paper. 


\section{References}

[1] Deck, R.T. (2010) A Logical Development of Quantum Mechanics from Physical Principles. CreateSpace 2010.

[2] Eisberg, R. and Resnick, R. (1985) Quantum Physics. 2nd Edition, John Wiley \& Sons, New York.

[3] Merzbacher, E. (1970) Quantum Mechanics. 2nd Edition, John Wiley \& Sons, New York.

[4] Liboff, R.L. (1992) Introductory Quantum Mechanics. 2nd Edition, Addison-Wesley, New York, 66-80.

[5] Dirac, P.A.M. (1958) The Principles of Quantum Mechanics. 4th Edition, Clarenden Press, Oxford.

[6] Goswami, A. (1992) Quantum Mechanics. 2nd Edition, Wm.C. Brown Publishers, Dubuque.

[7] Deck, R.T. and Ozturk, N. (1994) Foundations of Physics Letters, 7, 419-436. http://dx.doi.org/10.1007/BF02189245

[8] Sposito, G. (1970) An Introduction to Quantum Physics. John Wiley \& Sons, Inc., New York, 178. 